Regierungsübernahme und seine Umstände wieder verloren. Im Hinblick auf ihre allgemeine Wertschätzung sowie ihre wahrgenommene Glaubwürdigkeit und Problemlösungskompetenz ging die SPD mit ungünstigen Werten in den Wahlkampf. Der Spitzenkandidat konnte das nicht ausgleichen; zwar genoss Koch bei den Wählern keine höhere Wertschätzung als 2008, aber in der Kürze der verfügbaren Zeit konnte SchäferGümbel keine größere Popularität aufbauen. Die Meinungsumfragen ließen übereinstimmend eine klare Mehrheit für Schwarz-gelb und eine gravierende Niederlage für die Sozialdemokraten erwarten.

(4) Dem Desaster der misslungenen Regierungsbildung folgte für die SPD ein Desaster am Wahlabend, von dem aber nicht die Union, sondern FDP und Grüne profitieren konnten; auch der Linkspartei gelang der Wiedereinzug in den Landtag. Bei leicht gesunkener Wahlbeteiligung führte der Urnengang insgesamt zu einem Hessischen Landtag, der (gemessen über entsprechende Fraktionalisierungsindizes) zersplitterter ist denn je. Analysen der Hintergründe des Wahlergebnisses zeigen, dass der SPD alle Vorteile, die sich die Partei im Vorfeld der Wahl 2008 (etwa auf den Gebieten der Bildungs- und Klimapolitik, aber auch in der Gruppe der formal hoch gebildeten Wähler) erarbeitet hatte, wieder verloren gegangen waren. Hinzu kam ein starker negativer Effekt des Themas „Neuwahl“ selbst, der ebenfalls zu einer Abkehr vor allem von den Sozialdemokraten, aber auch von der CDU führte, die FDP und die Grünen jedoch stärkte.

(5) Nach raschen Koalitionsverhandlungen vereinbarten CDU und FDP ihre dritte Zusammenarbeit in Hessen. Roland Koch wurde wieder zum Ministerpräsidenten gewählt, wenngleich nicht mit allen Stimmen seiner Koalition. Als wichtige bundespolitische Implikation folgte aus der Bildung der CDU-FDP Regierung, dass die Große Koalition in Berlin künftig im Bundesrat keine eigene Mehrheit mehr hat, sondern auf die Zustimmung von Landesregierungen mit Beteiligung anderer Parteien angewiesen ist.

\title{
Parteimitgliedschaften im Jahre 2008
}

\author{
Oskar Niedermayer
}

Mit der folgenden Dokumentation wird der seit 2001 jährlich erscheinende systematische Überblick über die Entwicklung, regionale Verteilung und sozialstrukturelle Zusammensetzung der Parteimitgliedschaften fortgeschrieben. Zusätzliche Daten beziehungsweise Schaubilder sind über das Internet abrufbar (http://www.polsoz.fu-berlin.de/polwiss/forschung/ systeme/empsoz/schriften/Arbeitshefte/ahosz15.pdf). ${ }^{1}$ Es werden nur Daten aus den Mit-

1 Für eine Analyse dieser Daten sowie des Zeitraums vor der Vereinigung vgl. Oskar Niedermayer, Der Wandel des parteipolitischen Engagements der Bürger, in: Steffen Kühnel / ders. / Bettina Westle (Hrsg.), Wähler in Deutschland. Sozialer und politischer Wandel, Gender und Wahlverhalten, Wiesbaden 2008. 
gliederkarteien der Parteien verwendet (Stand jeweils 31. Dezember). ${ }^{2}$ Die Dokumentation der sozialstrukturellen Zusammensetzung der Parteimitgliedschaften enthält keine Angaben über die berufliche Stellung, weil die hierzu vorhandenen Daten den Beruf zum Zeitpunkt des Parteieintritts ohne spätere Aktualisierung wiedergeben und somit die tatsächliche Berufsstruktur der Parteimitgliedschaften nicht korrekt widerspiegeln.

Im Herbst 2008 wurde auf Anregung der Bundesgeschäftsführer vom Verfasser ein Workshop mit allen für die Mitgliederstatistik verantwortlichen Mitarbeitern der Parteien durchgeführt, auf dem eine Reihe von Verbesserungen der Statistik und ihrer Publikation beschlossen wurde. Daher gibt es in Zukunft eine Altersstatistik der Mitglieder mit einer identischen, sehr feinen Altersgruppeneinteilung, eine Statistik der Eintritte, Austritte und Todesfälle sowie der Eintritte nach Geschlecht und Alter. Die ersten dieser neuen Daten (noch nicht für alle Parteien) werden hier publiziert.

Trotz des Bemühens aller Parteien, ihre Mitgliedschaft wenigstens zu stabilisieren, mussten CDU, SPD und CSU im Jahre 2008 wieder Verluste hinnehmen. Am stärksten traf es, wie immer seit 2001, die SPD, aber auch die CDU, allerdings in geringerem Maße als in den letzten zwei Jahren. Bei der CSU hingegen verstärkte sich der Mitgliederverlust 2008 wieder. Die übrigen drei Parteien konnten ihre Mitgliederzahlen gegenüber 2007 jedoch erhöhen. FDP und Grüne konnten damit den Abwärtstrend der letzten zwei Jahre stoppen und die Linke erzielte einen erneuten Mitgliederzuwachs (vgl. Tabelle 1).

Während die beiden großen Parteien im Jahre 2007 in allen Bundesländern Mitglieder verloren hatten, kann die SPD 2008 in Brandenburg ein leichtes Plus verbuchen und bleibt in Mecklenburg-Vorpommern konstant; die CDU legt in Berlin, Brandenburg und Thüringen leicht zu. FDP und Grüne verzeichneten 2007 regional sehr unterschiedliche Mitgliederentwicklungen, und die Daten für 2008 zeigen dies auch, wobei der starke Mitgliederzuwachs der FDP in Bayern wohl auch der Mobilisierungswirkung durch das gute Abschneiden bei der Landtagswahl zuzuschreiben ist. Die Linke konnte 2008 in allen westdeutschen Ländern zulegen, musste aber in Ostdeutschland flächendeckend Verluste hinnehmen (vgl. Tabelle 2).

Betrachtet man die Rekrutierungsfähigkeit der Parteien (Parteimitglieder in Prozent der Parteibeitrittsberechtigten, das heißt der Bevölkerung ab 16 beziehungsweise 14 Jahren) nach Bundesländern im Jahre 2007 (für 2008 liegen noch keine Bevölkerungsdaten vor), so zeigt sich wie in den Vorjahren, dass bei der SPD und den Grünen die fünf ostdeutschen Bundesländer mit deutlichem Abstand zu allen westdeutschen Ländern das Schlusslicht bilden und bei der CDU nur die Stadtstaaten Berlin, Bremen und Hamburg ähnlich niedrige Rekrutierungsgrade aufweisen wie die ostdeutschen Länder. Bei der im Westen immer noch mitgliederschwachen FDP bestehen hingegen keine systematischen Ost-West-Unterschiede. Die Linke blieb auch 2007 eine ostdeutsche Regionalpartei, die im Westen - mit Ausnahme des Saarlands, der Heimat von Oskar Lafontaine - eine äußerst geringe Organisationsbasis aufweist. Aber auch im Osten erreichte sie nirgendwo auch nur annähernd die Rekrutierungsfähigkeit der SPD, CDU und CSU in ihren westdeutschen Hochburgen (vgl. Tabelle 3).

2 Den Geschäftsstellen der Parteien, die dem Verfasser die Daten freundlicherweise zur Verfügung gestellt haben, sei an dieser Stelle ausdrücklich gedankt. 
Beim Anteil der Frauen an den Parteimitgliedschaften ist über einen längeren Zeitraum hinweg kein parteiübergreifender Trend festzustellen. Während der Frauenanteil seit vielen Jahren bei der CDU leicht, bei der SPD und der CSU stärker ansteigt und sich bei den Grünen nach einem Absinken wieder nach oben bewegt hat, fällt er bei der FDP und der Linken (wobei sich der von der WASG kommende Zuwachs an Mitgliedern in einer deutlichen Verringerung des Frauenanteils 2007 gegenüber 2006 niederschlug). Zudem bestehen große Niveauunterschiede zwischen den Parteien: Die Frauen sind in der CSU am deutlichsten und in der Linkspartei immer noch am wenigsten unterrepräsentiert (vgl. Tabelle 4).

Betrachtet man die Entwicklung bis 2007, so scheint der bis etwa zur Jahrhundertwende zu beobachtende Rückgang des Anteils der jüngeren Parteimitglieder (bis 29/30 Jahre) vor allem für die SPD endgültig gestoppt zu sein, denn hier zeigte sich über mehrere Jahre hinweg eine leichte Verbesserung, während die Werte für die CDU und vor allem auch für die FDP in den letzten Jahren wieder abwärts gingen. In allen Parteien sind die Jüngeren jedoch immer noch deutlich unter- und die Älteren deutlich überrepräsentiert. Gravierend war die Überalterung bis 2006 vor allem bei der PDS. Der Zusammenschluss mit der WASG hat sich hier jedoch deutlich ausgewirkt: Sie hat 2007 bei den Jüngeren mit CDU, CSU und SPD gleichgezogen, weist allerdings immer noch den höchsten Anteil an Älteren (61 Jahre und älter) auf. Die 2007 zum ersten Mal vorhandenen Daten über die Altersstruktur der Grünen verdeutlichen, dass diese vor der FDP den höchsten Anteil an jüngeren Mitgliedern haben, aber auch dort sind die Jüngeren im Vergleich zu ihrem Anteil an der beitrittsberechtigten Bevölkerung noch deutlich unterrepräsentiert (vgl. Tabelle 5).

Die neue Alterstabelle für 2008 mit vollständiger Vergleichbarkeit und feinerer Gliederung zeigt, dass der Anteil der Mitglieder bis 20 Jahre bei CDU, SPD wie CSU sehr gering ist und deutlich unter dem der über 85-Jährigen liegt. Die Grünen weisen von den fünf Parteien mit 47 Jahren das geringste Durchschnittsalter auf, die SPD mit 58 Jahren das höchste, wobei die Daten für die Linke noch nicht vorliegen (vgl. Tabelle 6).

Für die CDU ist seit Mitte der 1990er Jahre ein kontinuierlicher Rückgang des Anteils konfessionsgebundener Mitglieder festzustellen, der sich auch 2008 fortgesetzt hat; für die CSU zeigt sich ein solcher eindeutiger Trend nicht (vgl. Tabelle 7). Der Katholikenanteil der CDU in Ostdeutschland ist zwar deutlich geringer als im Westen (vgl. Tabelle 8), setzt man ihn jedoch in Relation zum Anteil der Katholiken in der Bevölkerung, so zeigt sich, dass diese in der ostdeutschen CDU-Mitgliedschaft weit stärker (über-)repräsentiert sind als im Westen.

Die 2008 zum ersten Mal - bisher nur für CDU, SPD, CSU und FDP - zur Verfügung stehenden Daten über Eintritte, Austritte und Todesfälle im Laufe des Jahres belegen, dass die SPD in Relation zu ihrem Mitgliederbestand die geringste, die FDP die höchste Eintrittsquote und beide Parteien die höchste Austrittsquote aufwiesen. Zudem verloren die Parteien - je nach Grad der Überalterung ihrer Mitgliedschaft - 2008 zwischen 0,8 und 1,5 Prozent ihrer Mitglieder durch Todesfälle (vgl. Tabelle 9).

Der Frauenanteil bei den Eintritten liegt bei allen vier Parteien über dem Frauenanteil der Gesamtmitgliedschaft (vgl. Tabelle 10), und insgesamt sind die Neumitglieder im Vergleich zur Gesamtmitgliedschaft wesentlich jünger. Besonders deutlich zeigt sich dies bei der SPD: Während nur 3,4 Prozent aller Mitglieder bis 25 Jahre alt sind, beträgt der Anteil dieser Altersgruppe bei den Neumitgliedern des Jahres 2008 fast 32 Prozent (vgl. Tabelle 11). 


\begin{tabular}{|c|c|c|c|}
\hline \multirow{3}{*}{ 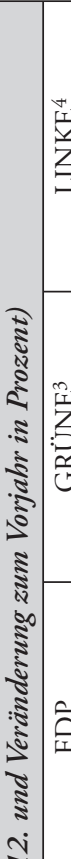 } & $t$ & 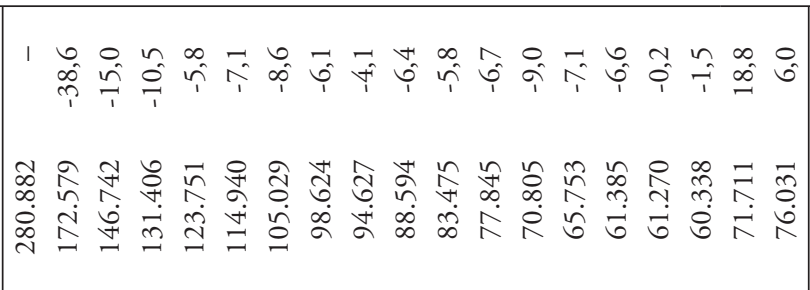 & \multirow{2}{*}{ 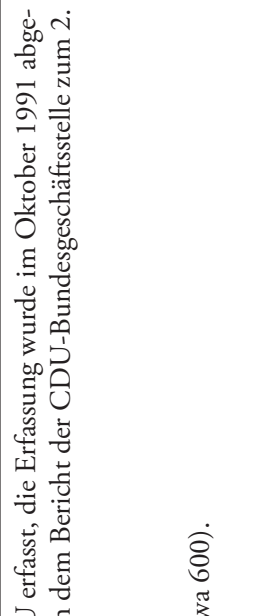 } \\
\hline & : & 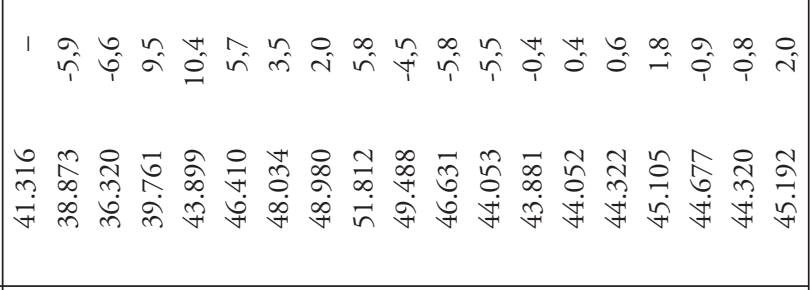 & \\
\hline & 它 & 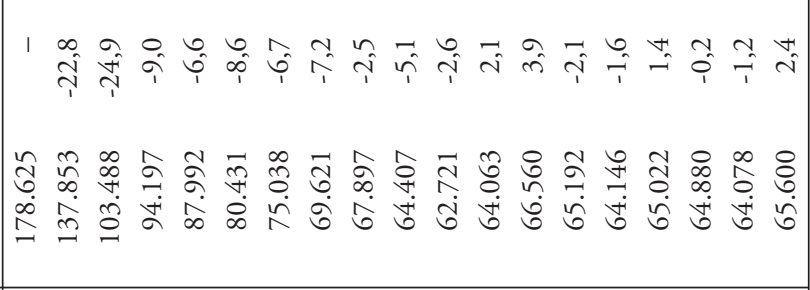 & 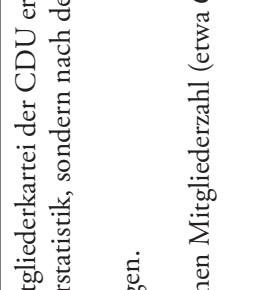 \\
\hline 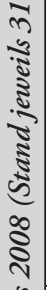 & $\vec{n}$ & 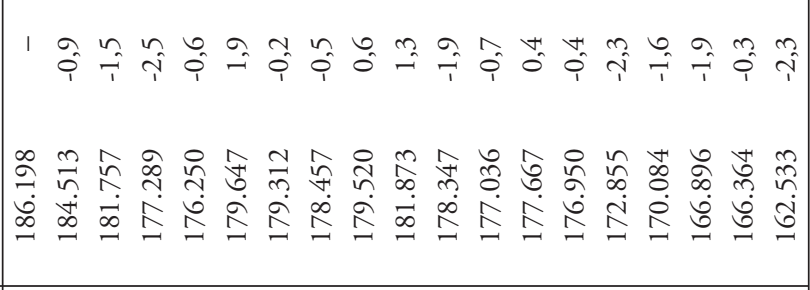 & 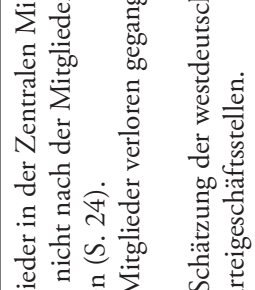 \\
\hline 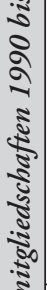 & فे & 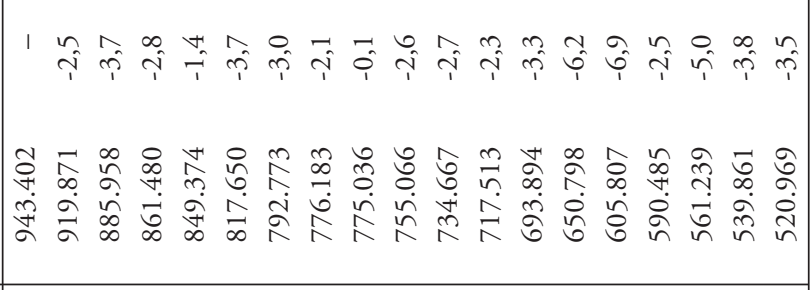 & 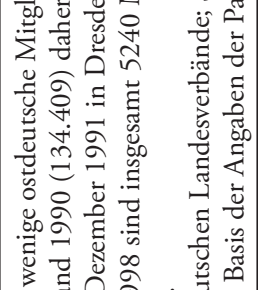 \\
\hline 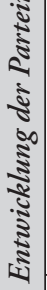 & $\overrightarrow{\mathrm{o}}$ & 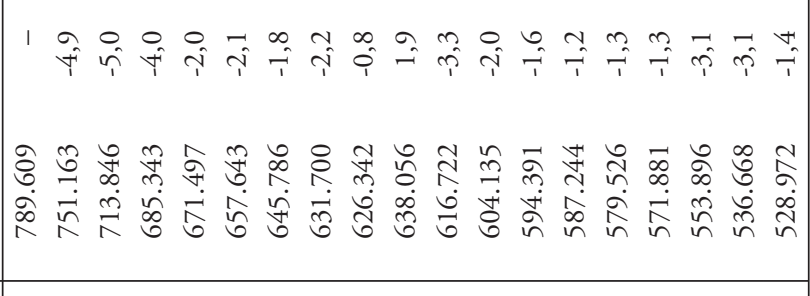 & 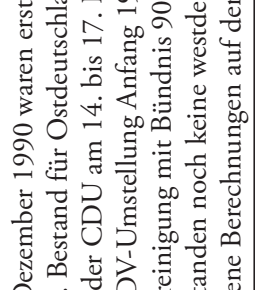 \\
\hline$\ddot{\sim}$ & & 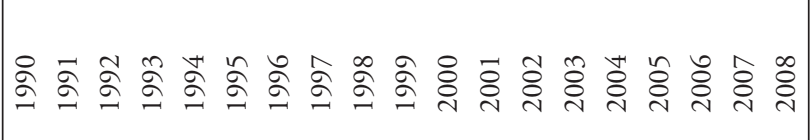 & 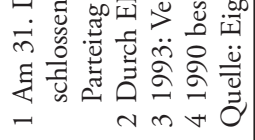 \\
\hline
\end{tabular}




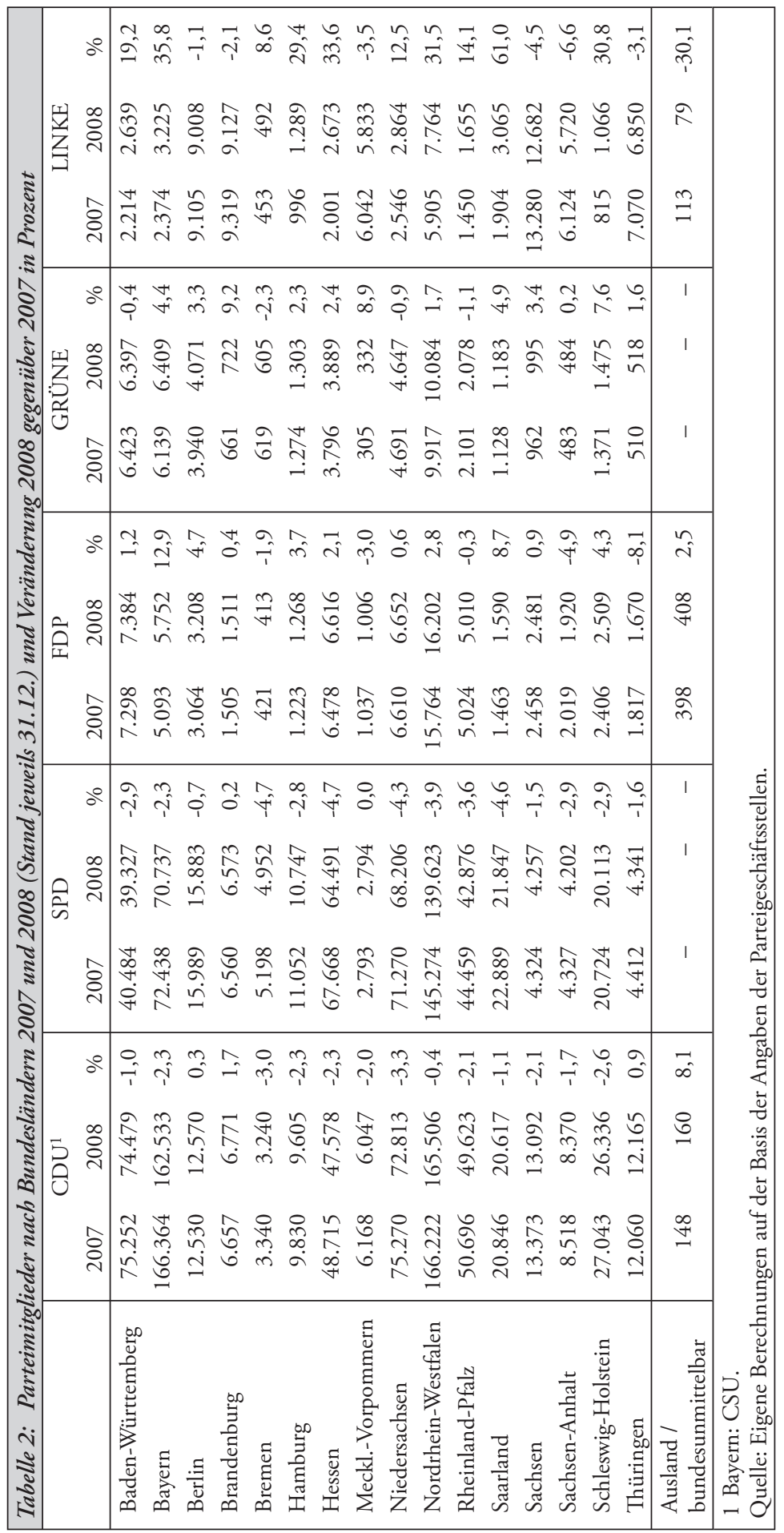




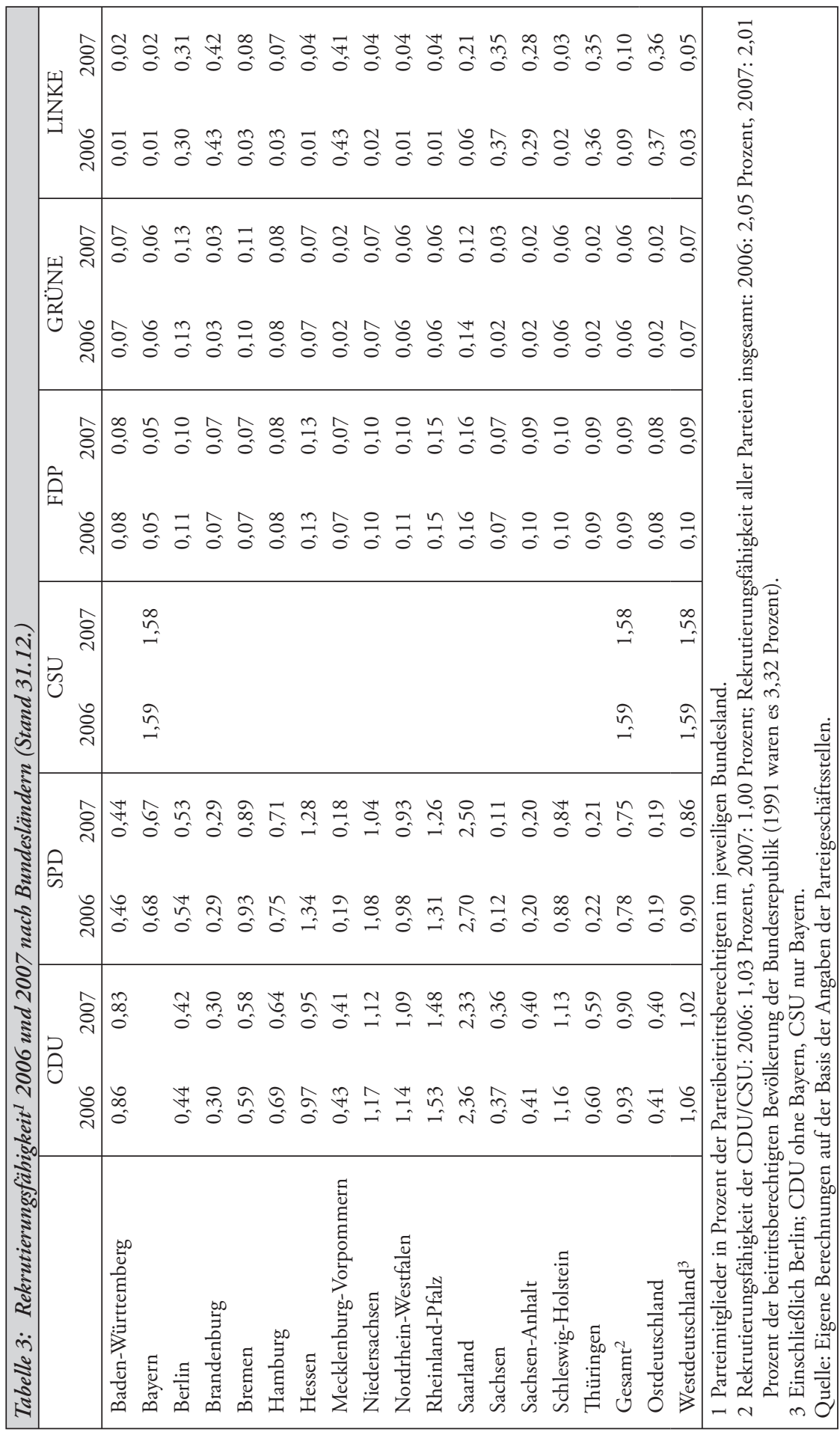




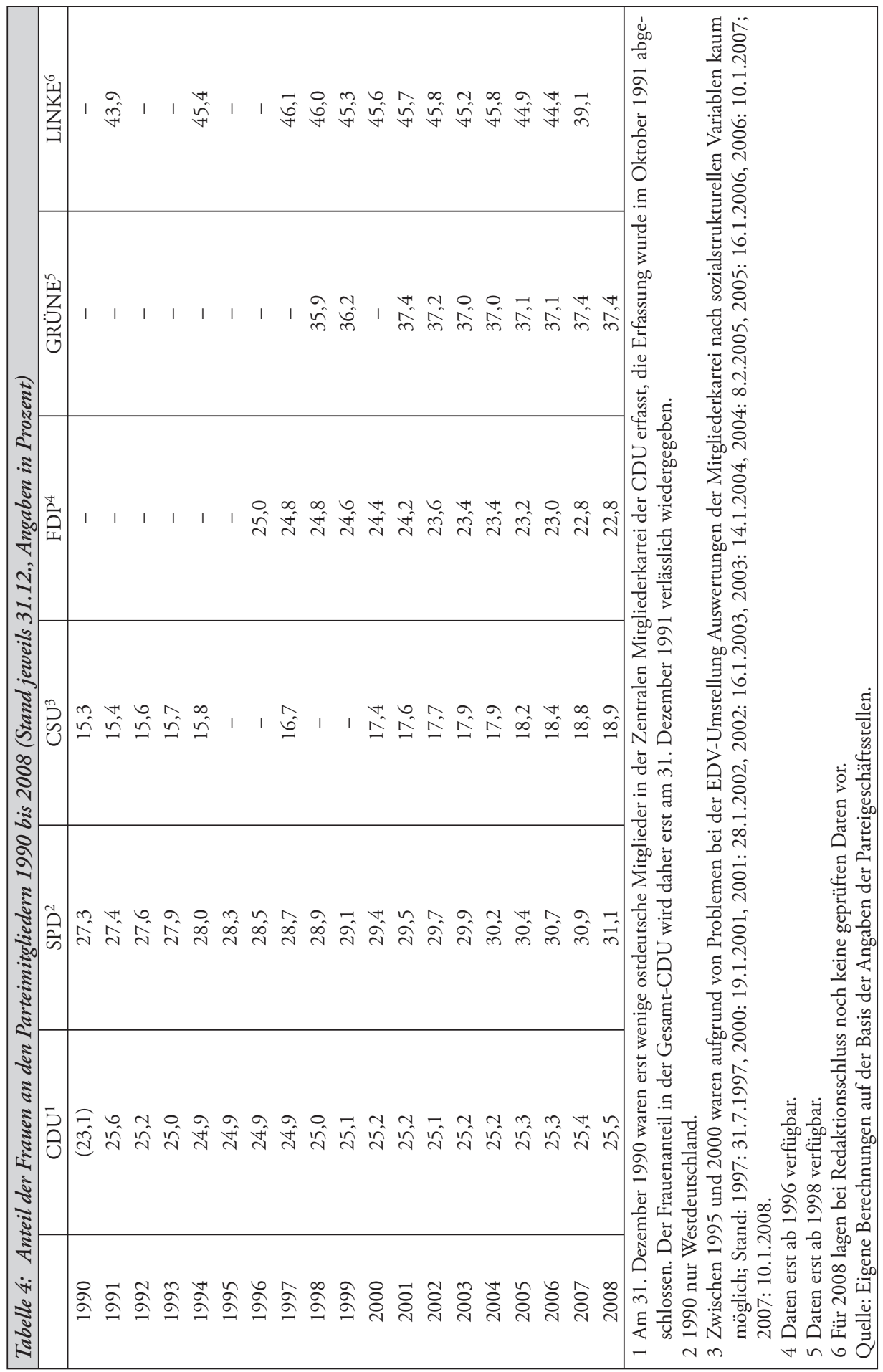




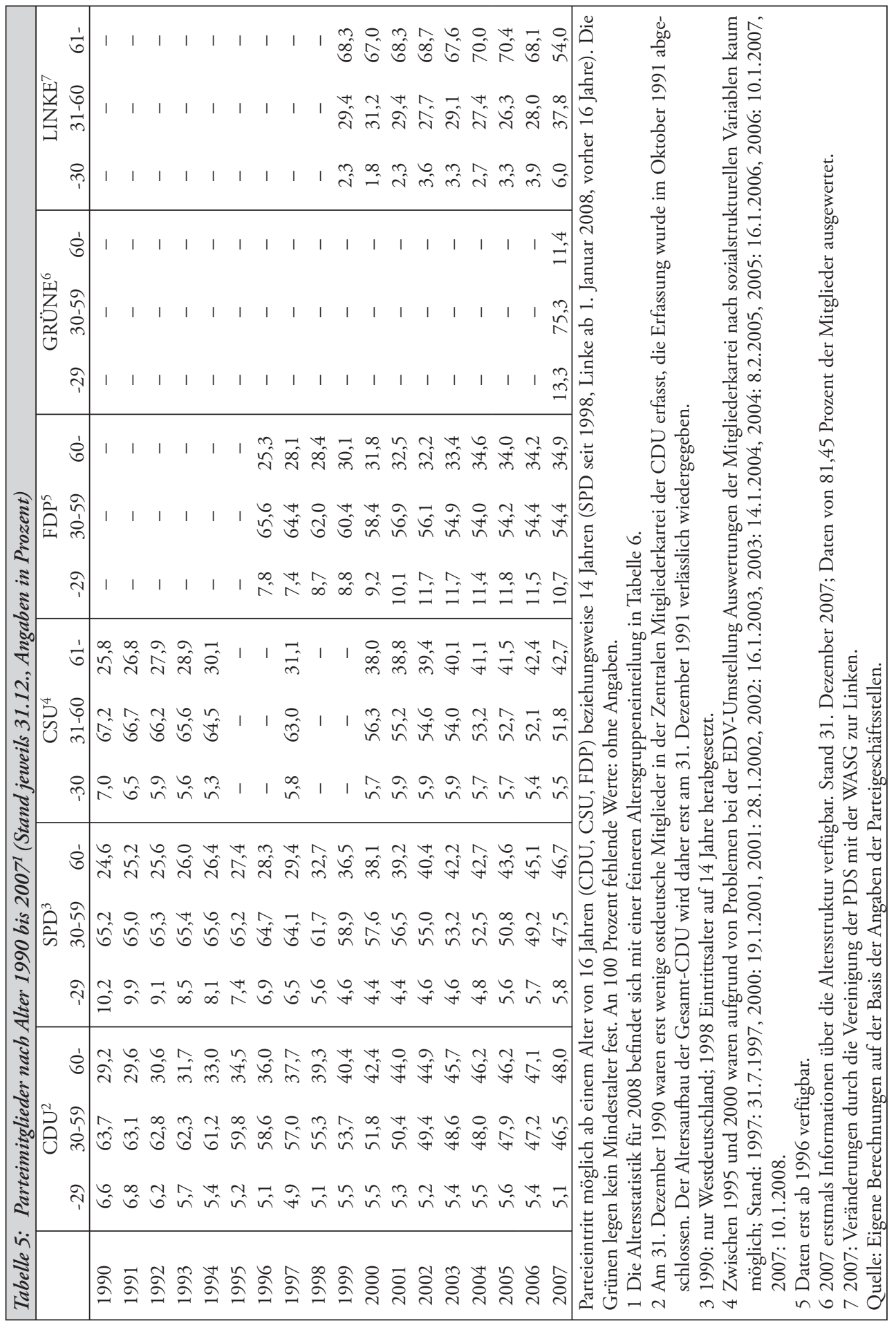




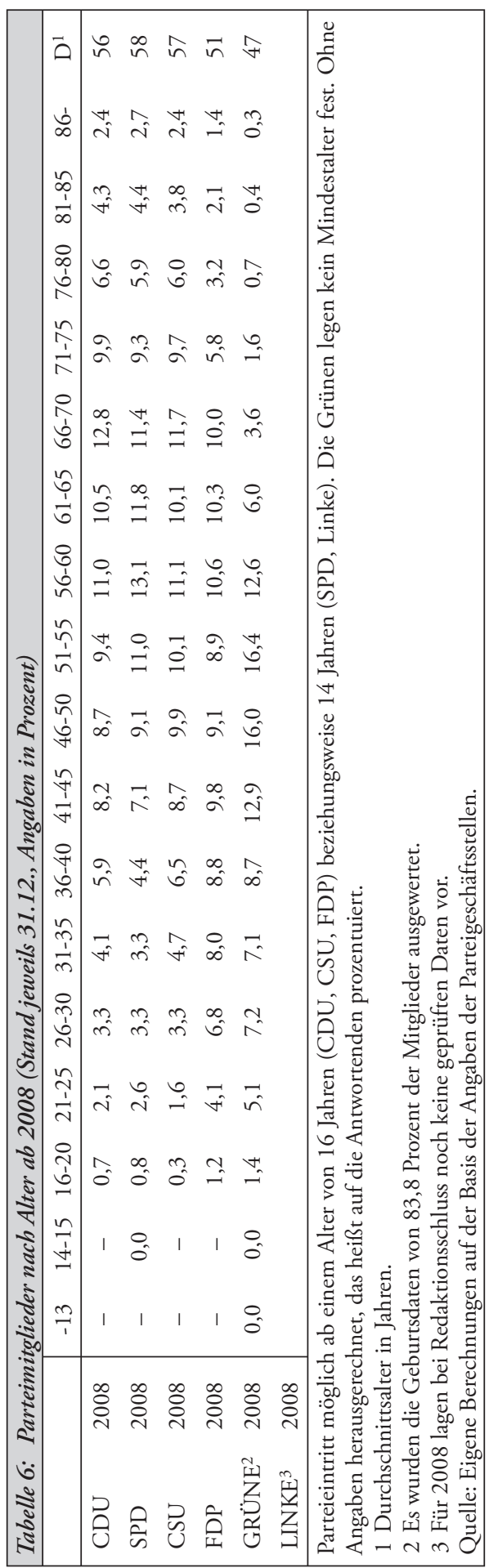




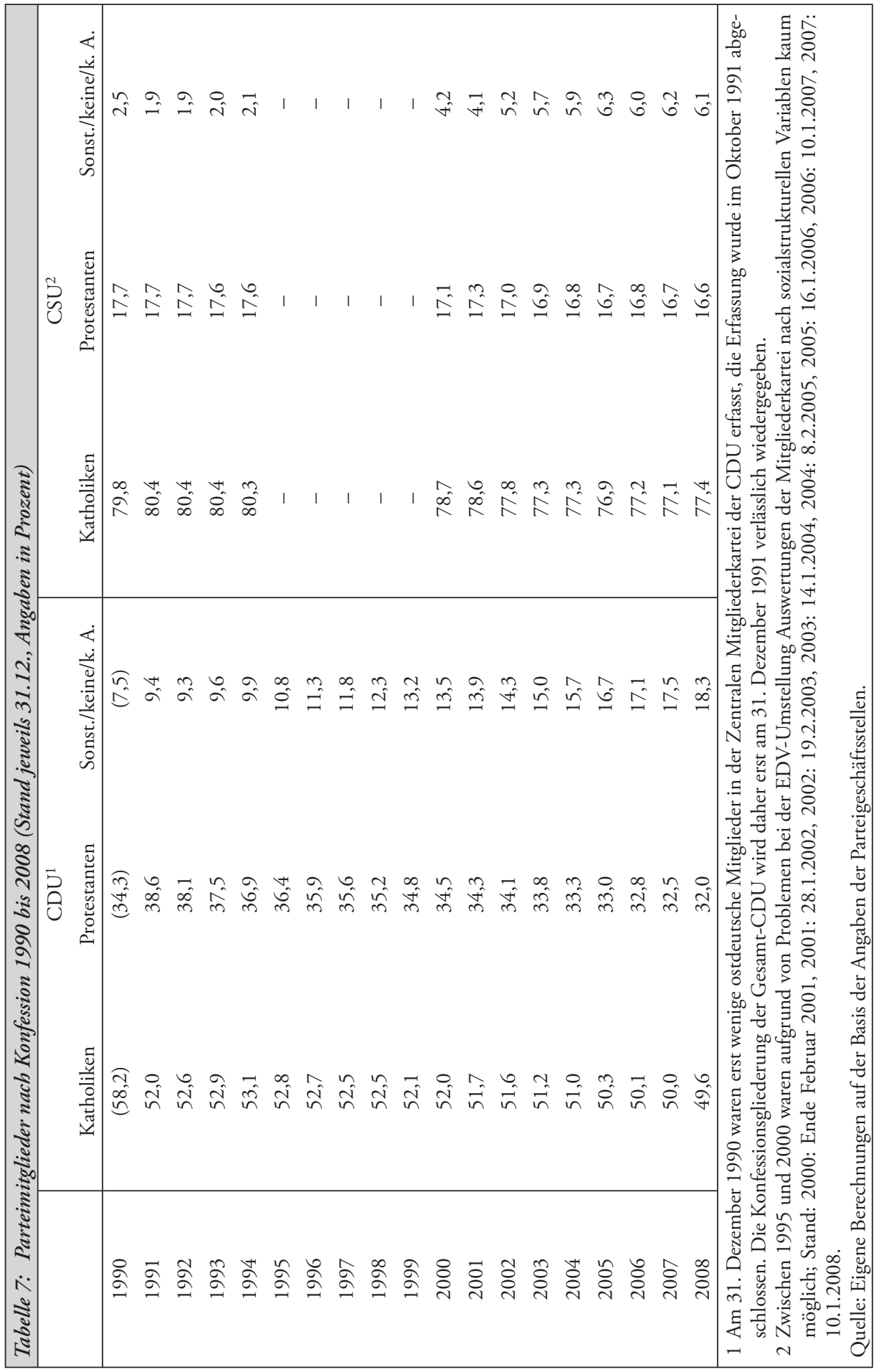




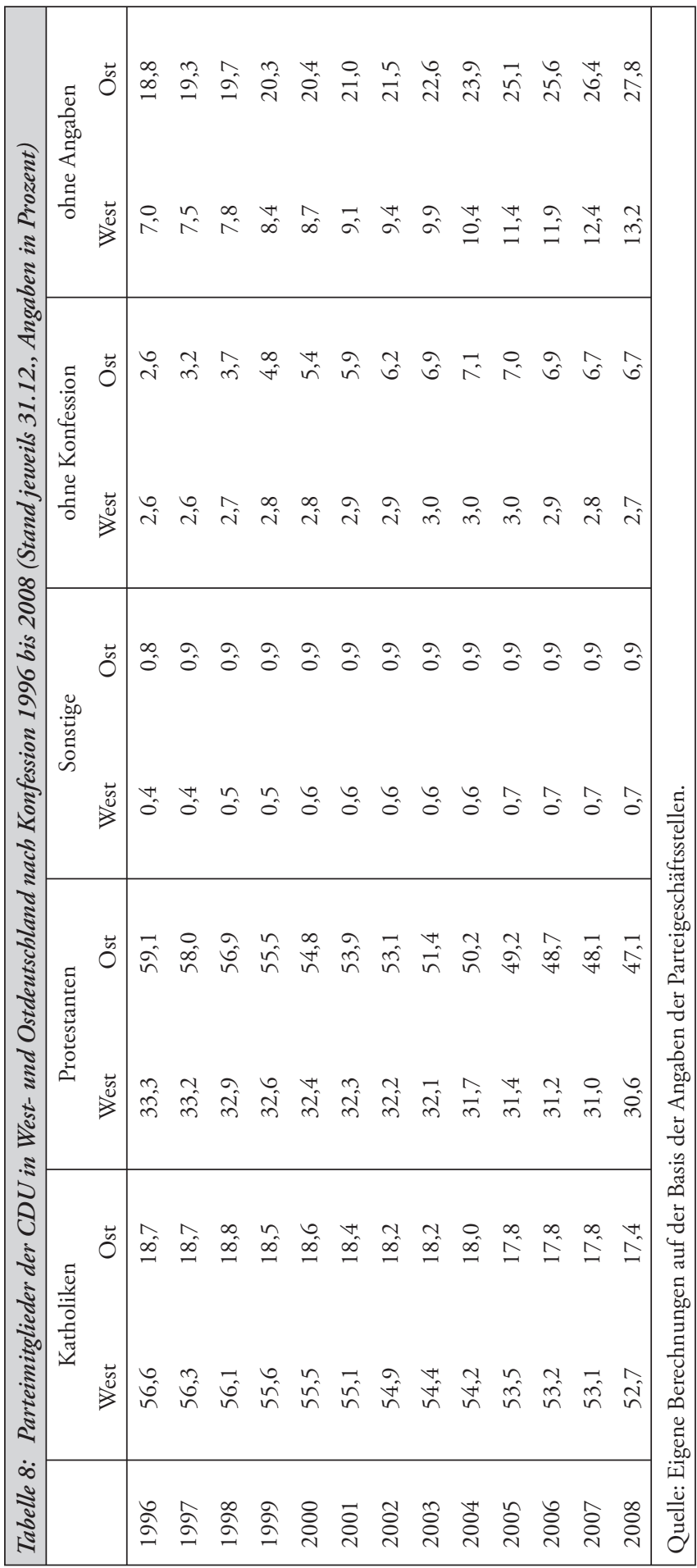




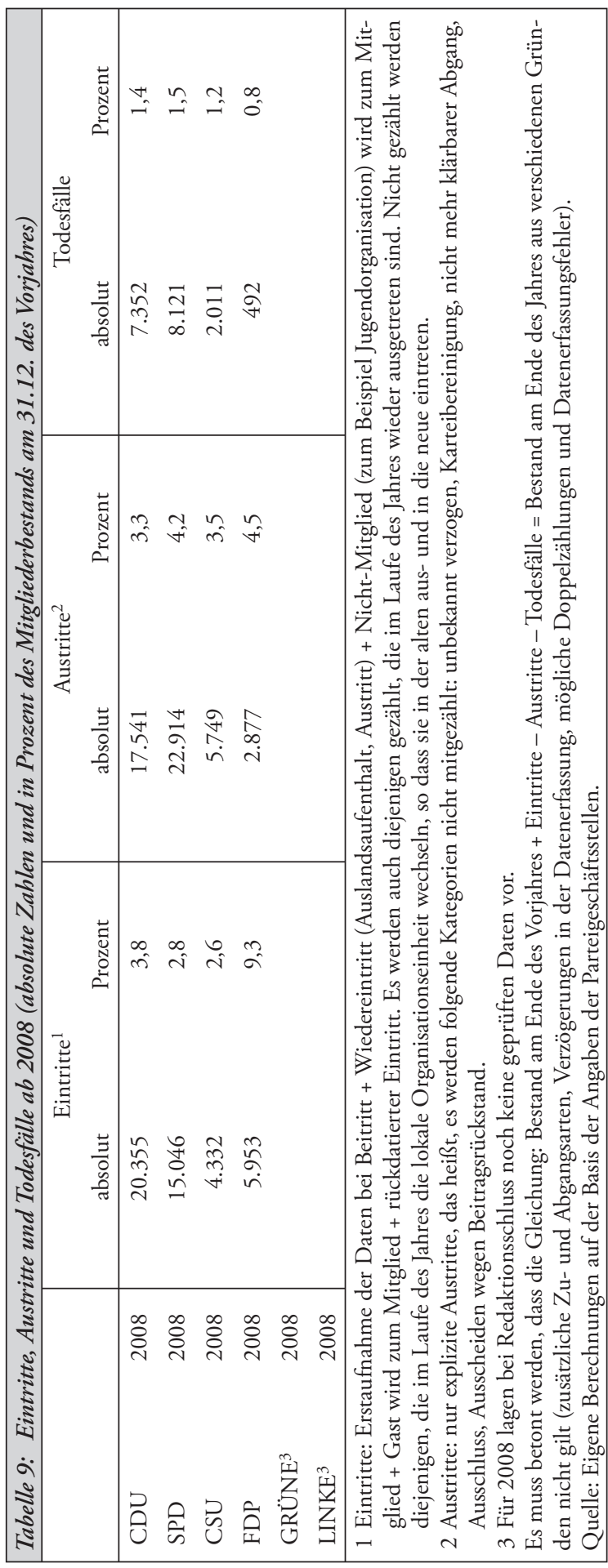




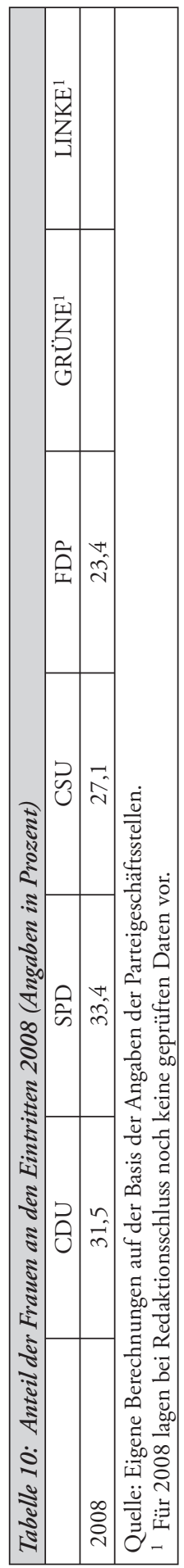

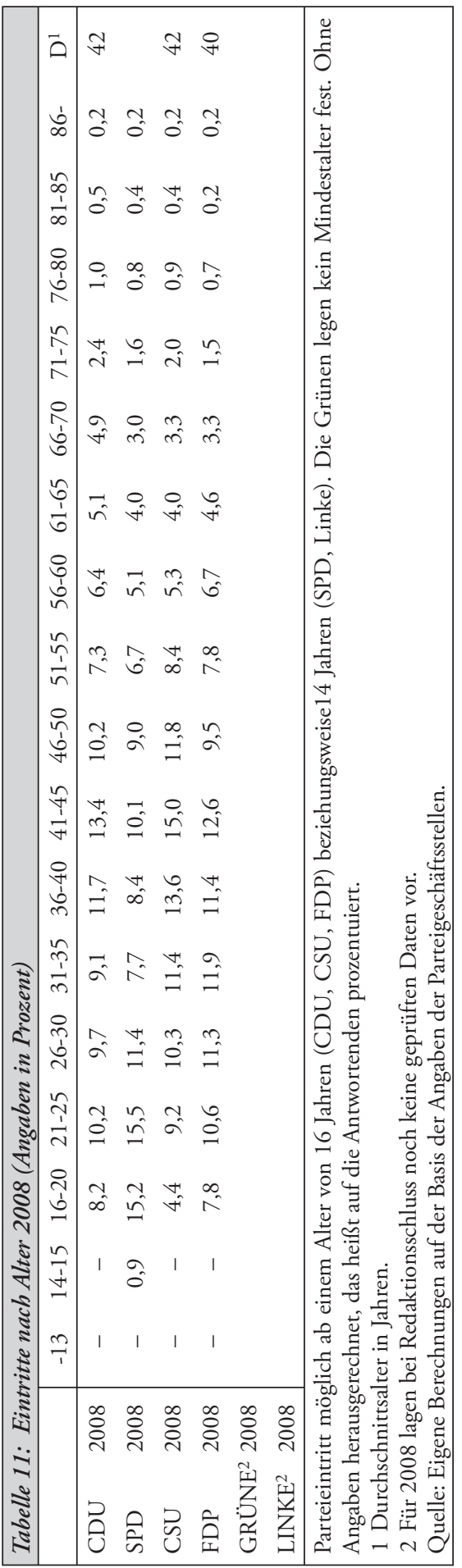

